\title{
REAPING THE BENEFITS OF INTERACTIVE SYNTAX AND SEMANTICS*
}

\author{
Kavi Mahesh \\ Georgia Institute of Technology \\ College of Computing \\ Atlanta, GA 30332-0280 USA \\ Internet: mahesh@cc.gatech.edu
}

\begin{abstract}
Semantic feedback is an important source of information that a parser could use to deal with local ambiguities in syntax. However, it is difficult to devise a systematic communication mechanism for interactive syntax and semantics. In this article, I propose a variant of left-corner parsing to define the points at which syntax and semantics should interact, an account of grammatical relations and thematic roles to define the content of the communication, and a conflict resolution strategy based on independent preferences from syntax and semantics. The resulting interactive model has been implemented in a program called COMPERE and shown to account for a wide variety of psycholinguistic data on structural and lexical ambiguities.
\end{abstract}

\section{INTRODUCTION}

The focus of investigation in language processing research has moved away from the issue of semantic feedback to syntactic processing primarily due to the difficulty of getting the communication between syntax and semantics to work in a clean and systematic way. However, it is unquestionable that semantics does in fact provide useful information which when fed back to syntax could help eliminate many an alternative syntactic structure. In this article, I address three issues in the communication mechanism between syntax and semantics and provide a complete and promising solution to the problem of interactive syntactic and semantic processing.

Since natural languages are replete with ambiguities at all levels, it appears intuitively that a processor with incremental interaction between the levels of syntax and semantics which makes the best and immediate use of both syntactic and semantic information to eliminate many alternatives would win over either a syntax-first or a semantics-first mechanism. In order to devise such an interactive mechanism, one has to address three important issues in the communication: (a) When to communicate: at what points should syntax and semantics interact, (b) What to communicate: what and how

*The author would like to thank his advisor Dr. Kurt Eiselt and his colleague Justin Peterson for their support and valuable comments on this work. much information should they exchange, and (c) How to agree: how to resolve any conflicting preferences between syntax and semantics.

In this article, I propose (a) a particular variant of left-corner parsing that I call Head-Signaled Left Corner Parsing (HSLC) to define the points where syntax and semantics should interact, (b) an account of grammatical relations based on thematic roles as a medium for communication, and (c) a simple strategy based on syntactic and semantic preferences for resolving conflicts in the communication. These solutions were motivated from an analysis of a large body of psycholinguistic data and account for a greater variety of experimental observations on how humans deal with structural and lexical ambiguities than previous models (Eiselt et al, 1993). While it also appears that the proposed interaction with semantics could make improvements to the efficiency of the parser in dealing with real texts, such a conclusion can only be drawn after an empirical evaluation.

\section{WHEN TO COMMUNICATE}

Syntax and semantics should interact only at those times when one can provide some information to the other to help reduce the number of choices being considered. Only when the parser has analyzed a unit that carries some part of the meaning of the sentence (such as a content word) can semantics provide useful feedback perhaps using selectional preferences for fillers of thematic roles. We need to design a parsing strategy that communicates with semantics precisely at such points. While pure bottom-up parsing turns out to be too circumspect for this purpose, pure top-down parsing is too eager since it makes its commitments too early for semantics to have a say. A combination strategy called Left Corner (LC) parsing is a good middle ground making expectations for required constituents from the leftmost unit of a phrase but waiting to see the left corner before committing to a bigger syntactic unit (E.g., Abney and Johnson, 1991). In LC parsing, the leftmost child (the left corner) of a phrase is analyzed bottom-up, the phrase is projected upward from the leftmost child, and other children of the phrase are projected top-down from the phrase. 
While LC parsing defines when to project topdown, it does not tell us when to make attachments. That is, it does not tell when to attempt to attach the phrase projected from its left corner to higher-level syntactic units. Should it be done immediately after the phrase has been formed from its left corner, or after the phrase is complete with all its children (both required and optional adjuncts), or at some intermediate point? Since ambiguities arise in making attachments and since semantics could help resolve such ambiguities, the points at which semantics can help, determine when the parser should attempt to make such attachments.

LC parsing defines a range of parsing strategies in the spectrum of parsing algorithms along the "eagerness" dimension (Abney and Johnson, 1991). The two ends of this dimension are pure bottomup (most circumspect) and pure top-down (most eager) parsers. Different LC parsers result from the choice of arc enumeration strategies employed in enumerating the nodes in a parse tree. In Arc Eager LC (AELC) Parsing, a node in the parse tree is linked to its parent without waiting to see all its children. Arc Standard LC (ASLC) Parsing, on the other hand, waits for all the children before making attachments. While this distinction vanishes for pure bottom-up or top-down parsing, it makes a big difference for LC Parsing.

In this work, I propose an intermediate point in the LC Parsing spectrum between ASLC and AELC strategies and argue that the proposed point, that 1 call Head-Signaled LC Parsing (HSLC), turns out to be the optimal strategy for interaction with semantics. In this strategy, a node is linked to its parent as soon as all the required children of the node are analyzed, without waiting for other optional children to the right. The required units are predefined syntactically for each phrase; they are not necessarily the same as the 'head' of the phrase. (E.g., $N$ is the required unit for NP, V for VP, and NP for PP.) HSLC makes the parser wait for required units before interacting with semantics but does not wait for optional adjuncts (such as PP adjuncts to NPs or VPs). The parsing spectrum now appears thus:

(Bottom-Up $\rightarrow$ Head-Driven $\rightarrow$ ASLC $\rightarrow$ HSLC

$\rightarrow$ AELC $\rightarrow$ Top-Down)

\section{Algorithm HSLC:}

Given a grammar and an empty set as the initial forest of parse trees,

For each word,

Add a new node $T_{w}$ to the current forest of trees $\left\{T_{i}\right\}$ for each category for the word in the lexicon mark $T_{w}$ as a complete subtree

Repeat until there are no more complete trees that can be attached to other trees,

Propose attachments for a complete subtree $T_{j}$

to a $T_{i}$ that is expecting $T_{j}$, or

to a $T_{i}$ as an optional constituent, or

to a new $T_{k}$ to be created if $T_{j}$ can be the left corner (leftmost child) of $T_{k}$

Select an attachment (see below) and attach

If a new $T_{k}$ was created, add it to the forest, and make expectations for required units of $T_{k}$

If a $T_{i}$ in the forest has seen all its required units,

Mark the $T_{i}$ as a complete subtree.

Consider a PP attachment ambiguity and the tree traversal labelings produced by different LC parsers shown in Figure 1. It can be seen from Figure la that AELC attempts to attach the PP to the VP or NP even before the noun in the PP has been seen. At this time, semantics cannot provide useful feedback since it has no information on the role filler for a thematic role to evaluate it against known selectional preferences for that role filler. Thus AELC is too eager for interactive semantics. ASLC, on the other hand, does not attempt to attach the VP to the $S$ until the very end (Fig 1b). Thus even the thematic role of the subject NP remains unresolved until the very end. ASLC is too circumspect for interactive semantics. HSLC on the other hand, attempts to make attachments at the right time for interaction with semantics (Fig lc).
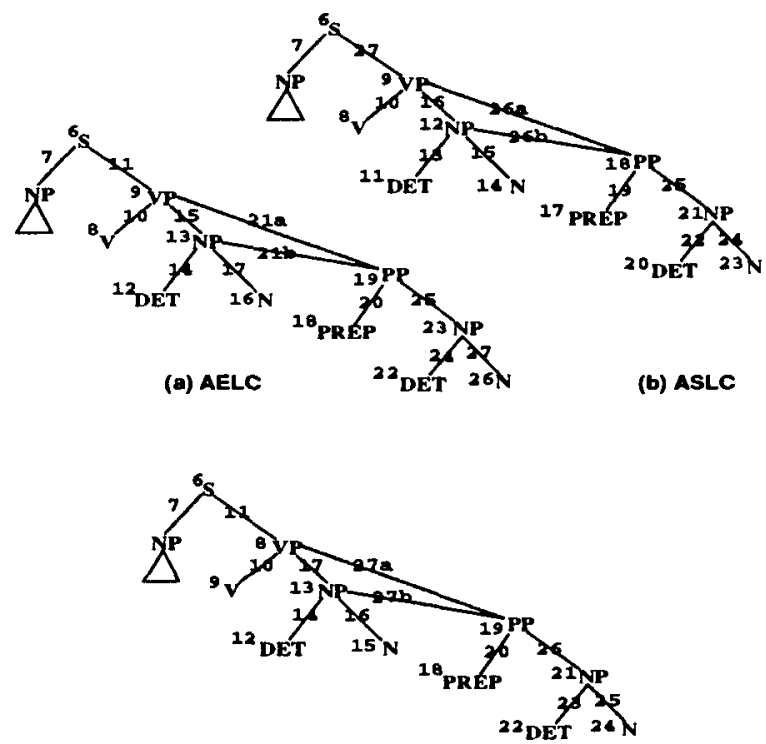

(c) HSLC

Figure 1: LC Parsers at an Attachment Ambiguity WHAT TO COMMUNICATE

The content of the communication between syntax and semantics is a set of grammatical relations and thematic roles. Syntax talks about the grammatical relations between the parts of a sentence such 
as Subject, Direct-object, Indirect-object, prepositional modifier, and so on. Semantics talks about the thematic relations between parts of the sentence such as event, agent, theme, experiencer, beneficiary, co-agent, and so on. These two closed classes of relations are translated to one another by introducing what I call "intermediate roles" to take into account other kinds of linguistic information such as active/passive voice, VP- vs. $\mathrm{NP}$-modification, and so on. Examples of intermediate roles are: active-subject, passive-subject, VP-With-modifier, subject-With-modifier, and so on. While space limitations do not permit a more detailed description here, the motivation for intermediate roles as declarative representations for syntax-semantics communication has been described in (Mahesh and Eiselt, to appear).

The grammatical relations proposed by syntax are translated to the corresponding thematic relations using the intermediate roles. Semantics evaluates the proposed role bindings using any selectional preferences for role fillers associated with the meanings of the words involved. It communicates back to syntax a set of either an Yes, a No, or a Don't-Care for each proposed syntactic attachment. A Yes answer is the result of satisfying one more selectional preferences for the role binding; a No for failing to meet a selectional constraint; and a Don't-Care when there are no known preferences for the particular role assignment.

\section{HOW TO AGREE}

Since syntax and semantics have independent preferences for multiple ways of composing the different parts of a sentence, an arbitrating process (that I call the Unified Process) manages the communication and resolves any conflicts. This unified process helps select the alternative that is best given the preferences of both syntax and semantics. In addition, since the decisions so made are never guaranteed to be correct, the unified process is not deterministic and has the capability of retaining unselected alternatives and recovering from any errors detected at later times. The details of such an error recovery mechanism are not presented here but can be found in (Eiselt et al, 1993) for example.

Syntax has several levels of preferences for the attachments it proposes based on the following criteria: Attachment (of a required unit) to an expecting unit has the highest preference. Attachment as an optional constituent to an existing (completed) unit has the next highest preference. Attachment to a node to be newly created (to start a new phrase) has the least amount of preference. These preferences are used to rank syntactic alternatives. The algorithm for the unified process:

Given: A set of feasible attachments $\left\{A_{i}\right\}$ where each $A_{i}$ is a list of the two syntactic nodes being attached, the level of syntactic preference, and one of (Yes, No,
Don't-Care) as the semantic feedback,

If the most preferred syntactic alternative has an Yes or Don't-Care, select it

else if no other syntactic alternative has a Yes, then select the most preferred syntactic alternative that has a Don't-Care

else delay the decision and pursue multiple interpretations in parallel until further information changes the balance.

\section{DISCUSSION}

The model of interactive syntactic and semantic processing proposed accounts for a wide range psycholinguistic phenomena related to the handling of lexical and structural ambiguities by human parsers. Its theory of communication and the arbitration mechanism can explain data that modular theories of syntax and semantics can explain as well as data that interactive theories can (Eiselt et al, 1993). For instance, it can explain why sentence (1) below is a garden-path but sentence (2) is not. (1) The officers taught at the academy were very demanding.

(2) The courses taught at the academy were very demanding.

HSLC is different from both head-driven parsing and head-corner parsing. It can be shown that the sequence of attachments proposed by HSLC is more optimal for interactive semantics than those produced by either of the above strategies. HSLC is a hybrid of left-corner and head-driven parsing strategies and exploits the advantages of both.

In conclusion, I have sketched briefly a solution to the three problems of synchronization, content, and conflict resolution in interactive syntax and semantics. This solution has been shown to have distinct advantages in explaining psychological data on human language processing. The model is also a promising strategy for improving the efficiency of syntactic analysis. However, the latter claim is yet to be evaluated empirically.

\section{REFERENCES}

Steven P. Abney and Mark Johnson. 1991. Memory Requirements and Local Ambiguities of Parsing Strategies. J. Psycholinguistic Research, $20(3): 233-250$.

Kurt P. Eiselt, Kavi Mahesh, and Jennifer K. Holbrook. 1993. Having Your Cake and Eating It Too: Autonomy and Interaction in a Model of Sentence Processing. Proc. Eleventh National Conference on Artificial Intelligence (AAAI-93), pp 380-385.

Kavi Mahesh and Kurt P. Eiselt. To appear. Uniform Representations for Syntax-Semantics Arbitration. To appear in Proc. Sixteenth Annual Conference of the Cognitive Science Society, Atlanta, GA, Aug 1994. 